\title{
Session 9: Infectious diseases II
}

\author{
Wednesday 31st October 2007. Moderator: Roberto Burioni
}

[09.00-09.30]

\author{
Bacterial targets for anti-infective antibodies \\ Eszter Nagy \\ Intercell AG, Vienna, Austria
}

\section{[09.30-09.50]}

An influenza N1 neuraminidase-specific monoclonal antibody with broad inactivating activity against $\mathrm{H} 5 \mathrm{~N} 1$ viruses

Vidadi Yusibov, Gene A. Palmer, Goran Orozovic, Robert Nardone, Patricia de la Rosa, Yoko Shoji and Vadim Mett

Fraunhofer USA Center for Molecular Biotechnology, 9 Innovation Way, Suite 200, Newark, DE 19711, USA

Influenza has a long history characterized by waves of pandemics and epidemics. Although flu epidemics occur nearly every year, pandemics fortunately do not occur very often. However, today we are faced with the potential of an influenza pandemic. An avian influenza virus of the type $\mathrm{H} 5 \mathrm{~N} 1$, currently causing an epidemic in poultry in Asia, is persistently spreading throughout the globe increasing the potential for outbreaks in human populations and perhaps leading to a pandemic. The virus is highly pathogenic in birds and humans and could potentially cause widespread illness and mortality. Several vaccine candidates based on potentially pandemic influenza strains and antiviral drugs have been tested in preclinical studies. The data obtained so far have shown some promise, but have also revealed some shortcomings of both approaches. We have identified and characterized an $\mathrm{H} 5 \mathrm{~N} 1$ neuraminidase-specific monoclonal antibody which specifically inhibited N1 neuraminidase activity of high pathogenic avian influenza strains from clades 1 and 2. This antibody could have potential for the treatment of oseltamivirand zanamivir-resistant strains of $\mathrm{H} 5 \mathrm{~N} 1$, and could be developed into a diagnostic tool.
[09.50-10.10]

Development of fully human Mabs neutralizing pathogenic orthopoxviruses

Nina Tikunova, Tatiana Yun, Ljudmila Shingarova*, Viktoria Voronina, Nikolai Bormotov, Vera Morozova, Eugenii Belanov, Mikhail Kirpichnikov* and Aleksandr Ilyichev State Research Center of Virology and Biotechnology "Vector", Koltsovo, Novosibirsk region, 630559, Russia

${ }^{*}$ Institute of bioorganic chemistry Russian Academy of Science, Moscow, 117997, Russia

One possible approach for development of therapeutic Mabs is selection of variable fragments of immunoglobulins from phage display libraries and then, combining them with human IgG constant regions. This approach was used for development of fully human Mabs able to neutralize orthopoxviruses including pathogenic orthopoxviruses.

Several tens of human scFvs against orthopoxviruses were selected from both synthetic Nissim library and a new immune library based on $\mathrm{Vh}$ and $\mathrm{Vl}$ genes cloned from the peripheral lymphocytes of vaccinia virus immune donors. The libraries were panned against live variola viruses (major and minor alastrim strains) and vaccinia virus. The selected $\mathrm{scFvs}$ were tested in ELISA experiments for their binding with vaccinia, cowpox, ectromelia viruses and several strains of variola virus. A standard assay of virus neutralization as the ability of antibodies to inhibit viral plaque formation in cells monolayer was performed with vaccinia, cowpox, monkeypox and variola viruses, and several scFvs showed neutralizing activities against the orthopoxviruses. Western-blot analysis of the orthopoxvirus proteins was performed for all neutralizing scFvs. Some scFvs developed p35 orthopoxvirus protein, while others did not bind any of the orthopoxvirus proteins. 
Several the most promising scFvs were then engineered to the whole IgG1 human molecules. These fully human Mabs were produced in HEK293T cells cotransfected with the pairs of plasmids bearing the genes encoding heavy and light chains. After purification by affine chromatography, ELISA and Western-blot were repeated to confirm the properties of the engineered fully human Mabs. The affinity constants of the Mabs were shown to be 50-200 times higher than for the parental scFvs. Some of the engineered fully human Mabs retained their ability to neutralize pathogenic orthopoxviruses.

[10.10-10.30]

[Review Presentation]

Marked enhancement of weak neutralizing activities of IgG1 mAbs specific for tetanus toxin in vivo by the IgG4 mAbs with almost nonexistent neutralizing activity against the toxin

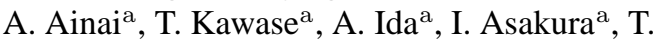
Kashimada $^{\mathrm{a}}$, M. Takahashi ${ }^{\mathrm{b}}$, I. Ishida ${ }^{\mathrm{c}}$ and J. Chiba ${ }^{\mathrm{a}, *}$ ${ }^{\mathrm{a} D e p a r t m e n t ~ o f ~ B i o l o g i c a l ~ S c i e n c e ~ a n d ~ T e c h n o l o g y, ~}$ Tokyo University of Science, 2641 Yamazaki, Noda, Chiba 278-8510, Japan

${ }^{\mathrm{b}}$ Department of Bacterial Pathogenesis and Infection Control, National Institute for Infectious Disease, Japan

${ }^{\mathrm{c}}$ Pharmaceutical Division, Kirin Brewery Co., Japan

${ }^{*}$ Corresponding author. E-mail: chibaj@rs.noda.tus. ac.jp.
We have generated tetroma cell lines by the fusion of newly developed parental 6JC5.2 triomas with a human B cell line, which was transformed with Epstein-Barr virus (EBV) and enriched with antibody-forming cells that produce neutralizing antibodies to tetanus toxin (TT) by a limiting dilution method using IL-6. The resultant two tetroma cell lines stably produced different monoclonal antibodies (mAbs), TT1 $(\operatorname{IgG} 1 \cdot \lambda)$ and TT2 $(\mathrm{IgG} 4 \cdot \kappa)$ reactive with TT after three-times consecutive cell cloning. Moreover, functional immunoglobulin $(\mathrm{Ig})$ genes were cloned from at least 10 cells in the first cloning step of tetromas after the cell fusion. None of the endogenous Ig genes, derived from the parental cell that hinders functional Ig gene cloning, was amplified. Although weak to almost nonexistent neutralizing activities against TT were detected in TT1 and TT2 mAbs, respectively, mixing of them resulted in a dramatic increase in the neutralizing activity and complete protection from the toxin was observed in vivo. The dramatic enhancement in the neutralizing activity was also observed when the several of different antiTT IgG1 mAbs that had been produced by KM mouse were mixed with TT2 mAbs. This classical but revised EBV-hybridoma method using fusion partner 6JC5.2 may become one alternative method for production of fully human antibodies selected by the intact human immune system and useful for prevention and treatment of infectious diseases and cancer. 